\title{
Heartbeat: is medical therapy for calcific aortic stenosis possible?
}

doi:10.1136/heartjnl-2020-318525

Catherine M Otto

There have been a proliferation of data on management of patients with severe calcific aortic stenosis (AS) over the past decade. But, no matter how effective, safe and durable valve replacement turns out to be, we still are treating (or mitigating) only the end-stage of a lengthy disease process. Success in treating calcific AS should be defined as the ability to slow haemodynamic progression or, ultimately, entirely prevent disease in the valve leaflets. In this issue of Heart, Lee and colleagues ${ }^{1}$ present intriguing data on the association between treatment with a dipeptidyl peptidase-4 (DPP-4) inhibitor and haemodynamic progression of AS in 212 patients (mean age about 73 years) with diabetes and mild-to-moderate AS. Patients taking a DPP-4 inhibitors with a potential favourable anti-calcification ability (such as linagliptin or gemigliptin), compared with those taking an unfavourable DPP-4 inhibitor (such as alogliptin, sitagliptin, or vildagliptin), had a smaller change in aortic velocity and less progression to severe AS ( $7.1 \%$ vs $29 \%, \mathrm{P}-0.03)$ with an HR of 0.116 (95\% CI 0.024 to $0.551, \mathrm{p}=0.007$ ) on Cox regression analysis after adjustment for age, baseline renal function and AS severity (figure 1).

Bing and Dweck ${ }^{2}$ discuss the strengths and limitations of this study in an editorial and put these findings into the context of shared mechanisms between calcific AS and atherosclerosis, hypertension and osteoporosis, as well as diabetes (figure 2). Bing and Dweck ${ }^{2}$ emphasise that observational association studies, such as the study by Lee and colleagues, ${ }^{1}$ are only hypothesis generating. 'Truth will out-but in the case of disease-modifying medical therapy for aortic stenosis, where effect sizes may be small and mechanisms complex, only after an adequately powered and wellconducted randomised controlled trial."

In a review article in this issue of Heart, San Román and colleagues ${ }^{3}$ re-examine the risk-benefit balance in a 'wait for symptoms' strategy for timing of valve replacement in asymptomatic patients with severe AS versus earlier intervention (figure 3). The potential role of risk markers is discussed and the ongoing

Correspondence to Professor Catherine M Otto, Division of Cardiology, University of Washington, Seattle, WA 98195, USA; cmotto@uw.edu

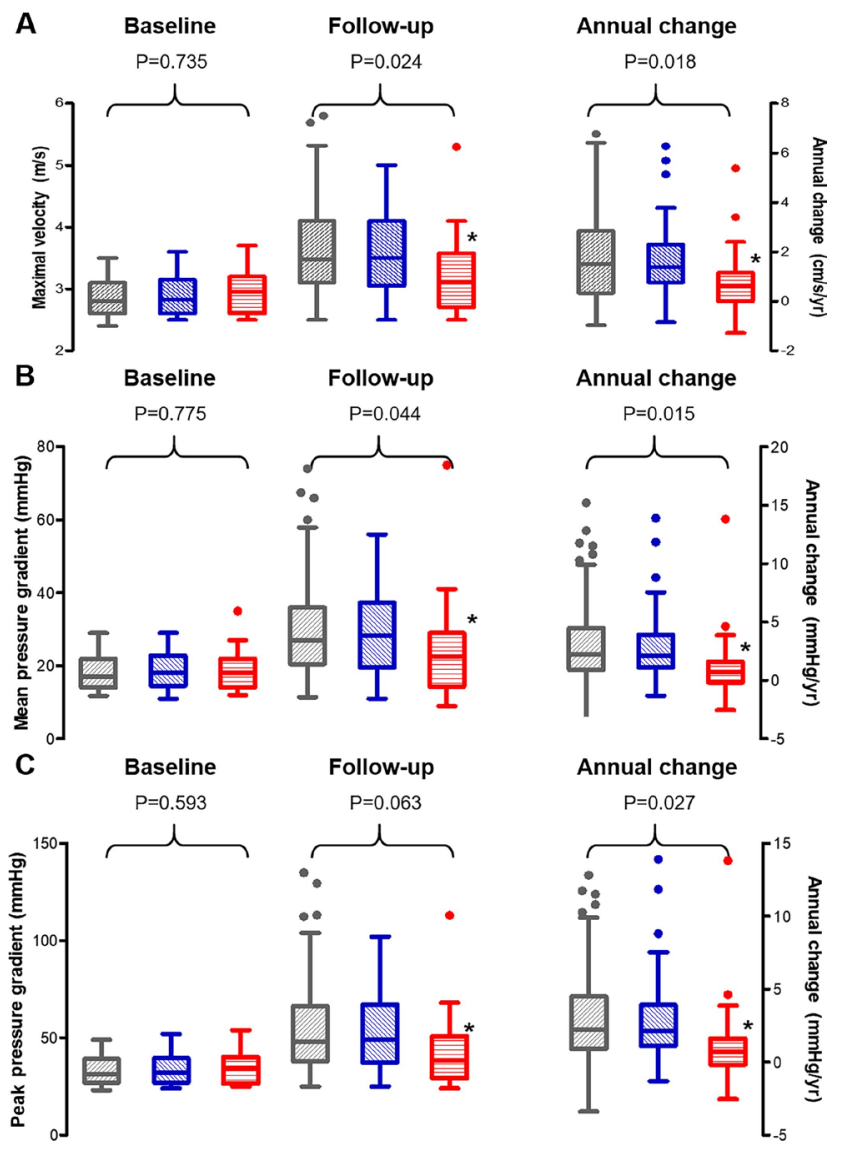

Box-and-Whiskers plot with Tukey's method

Box-and-Whiskers plot with Tukey's method
Nonuser Unfavourable DPP-4 inhibitor user Favourable DPP-4 inhibitor user ${ }^{*} p<0.05$ for the pairwise post-hoc comparison of Nonuser versus DPP-4 inhibitor user

Figure 1 Changes of maximal transaortic valve velocity (A), mean (B) and peak (C) pressure gradient according to medications. Turkey's method was used to make box plots. DPP-4, dipeptidyl peptidase-4.

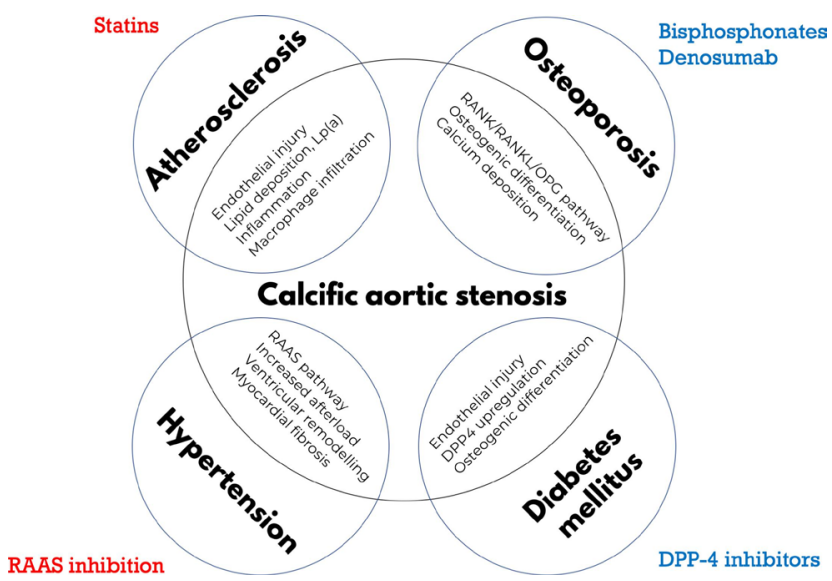

Figure 2 Schematic of proposed shared mechanisms between calcific aortic stenosis and other pathologies which have been investigated in, or are the current target of, clinical studies. Adapted from Dweck et al. ${ }^{10}$ DPP-4, dipeptidyl peptidase-4; Lp(a), lipoprotein (a); OPG, osteoprotegerin; RAAS, renin-angiotensin-aldosterone; RANKL, receptor activator of nuclear factor- $\kappa \mathrm{B}$ ligand. 


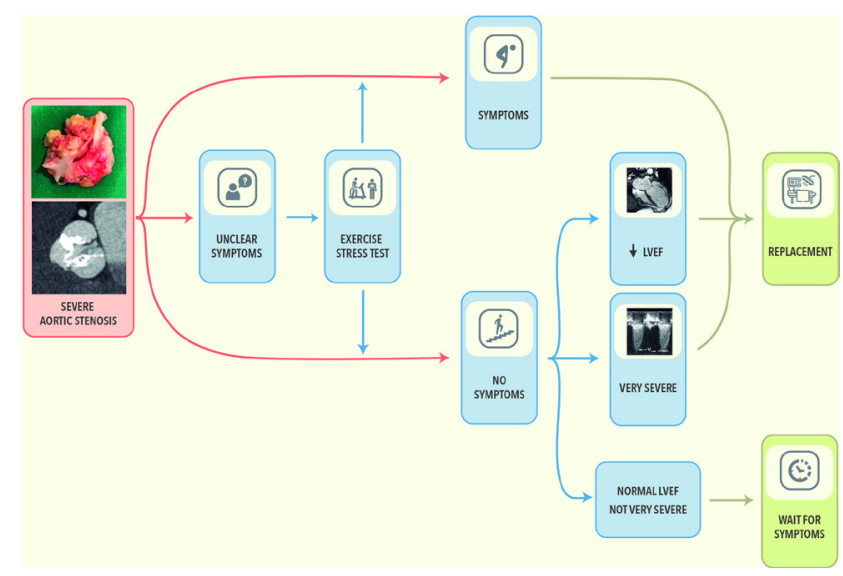

Figure 3 Management of a patient with asymptomatic severe aortic stenosis based on the evidence available. It could change if the ongoing randomised studies demonstrate that aortic valve replacement is better than the 'wait for symptoms' approach in terms of mortality or if the 'individualised strategy' shows to be of benefit (see text). Pictograms freely available at www. flaticon.com and humanpictogram2.0. LVEF, left ventricular ejection fraction.
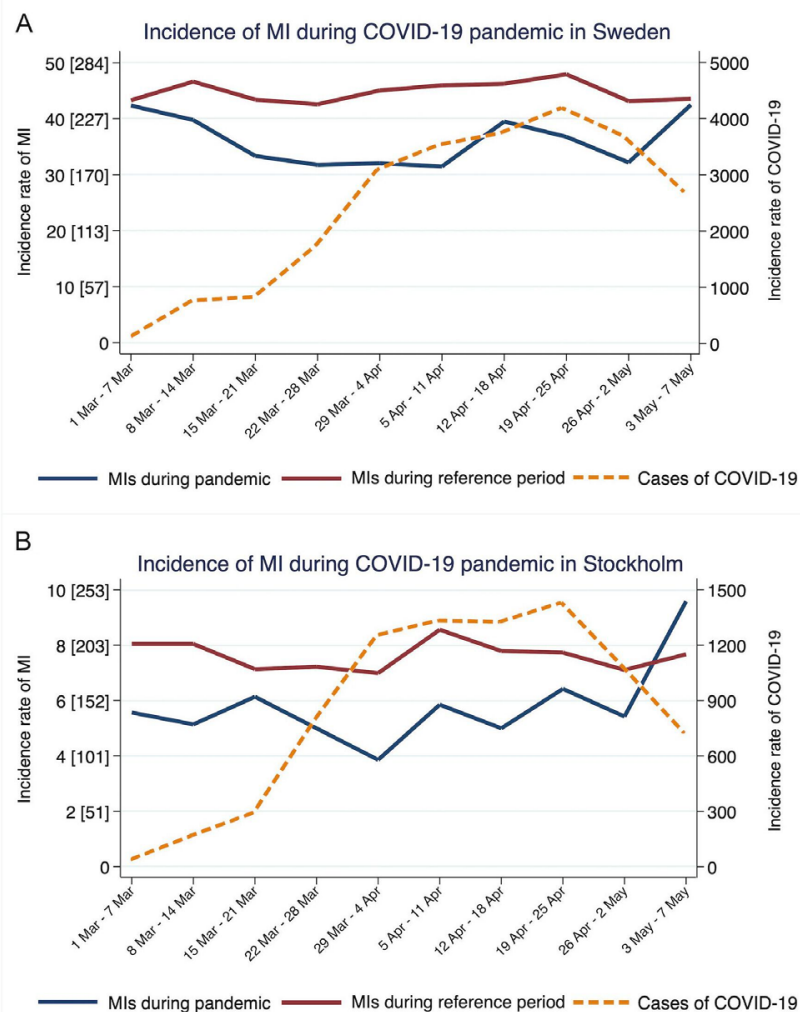

Figure 4 Incidence rate of myocardial infarction (MI) interventions and COVID-19 in Sweden as well as its capital city Stockholm. (A) Visualises the incidence rate of MI for each 7-day period during COVID-19 pandemic (1 March-May 2020) and the reference period (1 March 1-7 May, the years 2015-2019) together with the incidence of COVID-19 in Sweden. The incidence of MI is presented as daily incidence (absolute numbers) and the incidence rate per 100000 inhabitants per year in brackets. (B) Visualised the same information but for Stockholm county. A clear decline in $\mathrm{MI}$ incidence can be observed since the beginning of the pandemic both nationwide and isolated to Stockholm. On 12 April, a national campaign was launched throughout major newspapers, television channels, on the web and social media, aimed to inform and encourage patients with symptoms suggestive of $\mathrm{MI}$ to seek medical care. The inflow of patients with $\mathrm{MI}$ returned to typical levels both nationally as well as in Stockholm by 7 May 2020 reflecting how adequate countermeasures can reverse the indirect effects of COVID-19 pandemic on healthcareseeking behaviour. clinical trials addressing this timely question are summarised.

The impact of the COVID-19 pandemic on patients with cardiovascular disease was studied in two original research papers in this issue of Heart. Mohammad and colleagues $^{4}$ found a reduced incidence of patients diagnosed with myocardial infarction (MI) during the COVID-19 pandemic in Sweden with an incidence rate ratio of 0.80 (95\% CI 0.74 to $0.86, \mathrm{p}<0.001$ ) compared with 2015-2019. However, in those who did present for medical care, there was no change in referral for percutaneous coronary intervention (PCI) and no change in short-term mortality (figure 4). Bing and Adamson' 5 comment that 'Lower incidences of hospital admissions and invasive management of acute coronary syndromes are concerning and raise the spectre of excess morbidity and mortality due to delayed or absent provision of therapies.'

Similarly, in a study from the UK, Kwok and colleague 6 observed a $43 \%$ decline in PCI procedures in April 2020 compared with monthly averages over the preceding 2 years. Despite a longer interval from symptom onset to presentation and a slower door-to-balloon time, there was no difference for in-hospital mortality or major adverse cardiovascular events. In considering these and other studies, De Filippo et $\mathrm{al}^{7}$ propose we need to intensify our systems of care for acute MI. 'Increasing patient awareness of serious symptoms and inviting them to seek medical care in any case through dedicated campaigns, strengthening the territorial network with access points able to perform an ECG and to be in touch with hub centres, potentiating remote medical programmes with a clear definition of the roles and responsibilities of the healthcare professionals involved, getting an 'on call' dedicated staff trained to scrub in with protective equipment in a reasonable time, and setting up dedicated rooms where patients can undergo an extensive evaluation for the infection at a later time, thus prioritising angiography, are among the cornerstones of an 'emergency plan' that should be conceived and be easily available should a second wave of infections occur.'

The Education in Heart article in this issue $^{8}$ presents a guide to risk prediction and counselling in women with congenital heart disease who wish to become or are pregnant. This detailed text and tables nicely summarise risk scores and patient management; clinicians caring for younger women with congenital heart disease will find this article an essential resource. 
The Cardiology in Focus article ${ }^{9}$ in this issue nicely complements the Education in Heart article $^{7}$ with a thoughtful discussion of how to best communicate risk and benefits to cardiology patients. Recchia and Freeman recommend 'avoid using words to convey likelihoods: use numbers, and support them with graphics wherever possible. Be upfront and as precise as possible about uncertainties (again, using numerical ranges rather than verbal cues of uncertainty where possible). Be as balanced as you can about both benefits and risks, and avoid framing the numbers in just one direction. Moreover, the best way to check whether you have been successful in your communication is to stop and ask the patient to explain back what they have understood: this gives you a chance to assess what they are understanding, as well as what is important to them.'

Funding The authors have not declared a specific grant for this research from any funding agency in the public, commercial or not-for-profit sectors.
Competing interests None declared.

Patient and public involvement Patients and/or the public were not involved in the design, or conduct, or reporting, or dissemination plans of this research.

Patient consent for publication Not required.

Provenance and peer review Commissioned; internally peer reviewed.

(c) Author(s) (or their employer(s)) 2020. No commercial re-use. See rights and permissions. Published by BMJ.

\section{Check for updates}

To cite Otto CM. Heart 2020;106:1783-1785.

Heart 2020;106:1783-1785.

doi:10.1136/heartjnl-2020-318525

\section{ORCID iD}

Catherine M Otto http://orcid.org/0000-0002-05279392

\section{REFERENCES}

1 Lee S, Lee S-A, Choi B, et al. Dipeptidyl peptidase-4 inhibition to prevent progression of calcific aortic stenosis. Heart 2020;106:1824-31.

2 Bing R, Dweck MR. The quest for an aortic stenosis cure. Heart 2020;106:1790-1.
3 San Román JA, Vilacosta I, Antunes MJ, et al. The 'wait for symptoms' strategy in asymptomatic severe aortic stenosis. Heart 2020;106:1792-7.

4 Mohammad MA, Koul S, Olivecrona GK, et al. Incidence and outcome of myocardial infarction treated with percutaneous coronary intervention during COVID-19 pandemic. Heart 2020;106:1812-8.

5 Bing R, Adamson PD. Cardiac catheterisation laboratory in a global pandemic: ceding centre stage. Heart 2020;106:1788-9.

6 Kwok CS, Gale CP, Kinnaird T, et al. Impact of COVID-19 on percutaneous coronary intervention for ST-elevation myocardial infarction. Heart 2020;106:1805-11.

7 De Filippo 0, D'Ascenzo F, Deferrari GM. COVID-19 pandemic and infarctions: another call to reorganise our healthcare systems. Heart 2020;106:1786-7.

8 van Hagen IM, Roos-Hesselink JW. Pregnancy in congenital heart disease: risk prediction and counselling. Heart 2020;106:1853-61.

9 Recchia G, Freeman A. Communicating risks and benefits to cardiology patients. Heart 2020;106:1862-3.

10 Dweck MR, Boon NA, Newby DE. Calcific aortic stenosis: a disease of the valve and the myocardium. J Am Coll Cardiol 2012;60:1854-63. 